INPLASY

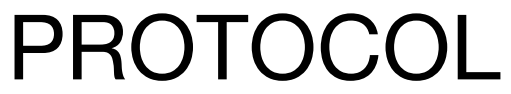

To cite: Gao et al. Association between prenatal phthalate exposure and gestational metabolic syndrome parameters: A systematic review of epidemiological study. Inplasy protocol 2020120065. doi: 10.37766/inplasy2020.12.0065

Received: 11 December 2020

Published: 12 December 2020

Corresponding author: Hui Gao

gh20190130@163.com

Author Affiliation:

The First Affiliated Hospital of Anhui Medical University

Support: Funding.

Review Stage at time of this submission: Data analysis.

Conflicts of interest:

None.

\section{Association between prenatal phthalate exposure and gestational metabolic syndrome parameters: A systematic review of epidemiological study}

Gao, $\mathrm{H}^{1}$; Zhang, C2; Tao, FB³.

Review question / Objective: Population:pregnant women. Exposure: all phthalates or phthalate metabolites exposure. Comparison: lower levels of exposure group, or different levels of target outcome group, or no target disease control group. Outcome: gestational diabetes, glucose, glycated haemoglobin, insulin, hypertensive disorder of pregnancy, hypertension, systolic blood pressure, diastolic blood pressure, gestational weight gain, total cholesterol, LDLcholesterol, HDL-cholesterol, triglycerides, metabolic syndrome. Study design: observational study, including cohort study, case-control study, cross-sectional survey.

INPLASY registration number: This protocol was registered with the International Platform of Registered Systematic Review and Meta-Analysis Protocols (INPLASY) on 12 December 2020 and was last updated on 12 December 2020 (registration number INPLASY2020120065).

\section{INTRODUCTION}

Review question / Objective: Population:pregnant women. Exposure: all phthalates or phthalate metabolites exposure. Comparison: lower levels of exposure group, or different levels of target outcome group, or no target disease control group. Outcome: gestational diabetes, glucose, glycated haemoglobin, insulin, hypertensive disorder of pregnancy, hypertension, systolic blood pressure, diastolic blood pressure, gestational weight gain, total cholesterol, LDL-cholesterol, HDL-cholesterol, triglycerides, metabolic syndrome. Study design: observational 
study, including cohort study, case-control study, cross-sectional survey.

Condition being studied: Fourteen studies were included in the present systematic review, which compromised 6 for blood pressure related indicators, 7 for glycemia related indicators and 2 for GWG. Two studies used one-spot serum sample for evaluation of phthalate exposure, while others used 1-4 urine samples. Concentrations of phthalate metabolites vary hugely among these publications, and the levels in serum were greatly lower than those in urine. These studies observed no inter- or intra-study consistency for any phthalate metabolite cross-sectionally or longitudinally associated with any of the indicators of HDCP, GDM or excessive GWG in pregnant women. Most reported associations were not significantly different from null result. Besides, positive and negative relationships also existed.

\section{METHODS}

Participant or population: Inclusion: pregnant women. Exclusion: not pregnancy status.

Intervention: Inclusion: all phthalates or phthalate metabolites exposure. Exclusion: not phthalate or its metabolite exposure.

Comparator: Inclusion: lower levels of exposure group, or different levels of target outcome group, or no target disease control group. Exclusion: other controls.

Study designs to be included: Inclusion: observational study, including cohort study, case-control study, cross-sectional survey. Exclusion: review, systematic review, metaanalysis, comment, etc.

Eligibility criteria: The inclusion and exclusion criteria following the PECOS (Population, Exposure, Comparison, Outcome, Study design) format were summarized in Supplemental Table S2. Inclusion criteria were the following: (日) Population: pregnant women; (月)Exposure: all phthalates or phthalate metabolites exposure; (火)Comparison: lower levels of exposure group, or different levels of target outcome group, or no target disease control group; (水)Outcome: gestational diabetes, glucose, glycated haemoglobin, insulin, hypertensive disorder of pregnancy, hypertension, systolic blood pressure, diastolic blood pressure, gestational weight gain, total cholesterol, LDL-cholesterol, HDL-cholesterol, triglycerides, metabolic syndrome; (木) Study design: all observational studies including cohort study, case-control study and crosssectional survey.

Information sources: The systematic literature search was performed in MEDLINE (accessed through PubMed), Web of Science, and CNKI (Chinese National Knowledge Infrastructure) until April 2020. The following search terms were used in combination: "phthalate" or "endocrine disruptor" and their variants, with the words "weight", "gestational weight gain", "insulin", "glycemia", "glucose", "Glycated Hemoglobin A", Hba1c, BMI, "body mass index", LDL, HDL, "cholesterol", "triglycerides", "plasma lipids", "blood lipids", "blood pressure", "gestational diabetes", "hypertension", "hypertensive disorder", "preeclapsia", "eclampsia", "metabolic syndrome", and the words "pregnancy", "in utero", "gestational" and their variants. The detailed search strategy list for each database was provided in Supplemental Table 1. We only considered human studies. Additional relevant studies were obtained by screening and searching references.

Main outcome(s): Fourteen studies were included in the present systematic review, which compromised 6 for blood pressure related indicators, 7 for glycemia related indicators and 2 for GWG. Two studies used one-spot serum sample for evaluation of phthalate exposure, while others used 1-4 urine samples. Concentrations of phthalate metabolites vary hugely among these publications, and the levels in serum were greatly lower than those in urine. These studies observed no inter- or intrastudy consistency for any phthalate 
metabolite cross-sectionally or longitudinally associated with any of the indicators of HDCP, GDM or excessive GWG in pregnant women. Most reported associations were not significantly different from null result. Besides, positive and negative relationships also existed.

Quality assessment / Risk of bias analysis: Of the 14 publications, the STROBE scores ranged from 10 to 19 . Seven $(50 \%), 6(43 \%)$, and $1(7 \%)$ studies were respectively evaluated as high, moderate, and low quality of evidence.

Strategy of data synthesis: Qualitative analysis.

Subgroup analysis: None.

Sensibility analysis: None.

Country(ies) involved: China.

Keywords: phthalate; prenatal exposure; gestational metabolic syndrome; systematic review.

Contributions of each author:

Author 1 - Hui Gao.

Author 2 - Cheng Zhang.

Author 3 - Fangbiao Tao. 